J. Phys. E: Sci. Instrum., Vol. 13, 1980. Printed in Great Britain

\section{Rate control in dual source evaporation}

T Wielinga, W Gruisinga, H Leeuwis, J C Lodder, $\mathbf{J} \mathbf{F} \vee$ Weers and $\mathbf{J} \mathbf{C}$ Wilmans

Department of Electrical Engineering, Twente University of Technology, 7500 AE Enschede, The Netherlands

\section{Received 10 April 1979, in final form 30 August 1979}

Abstract Two-component thin films are deposited in a high-vacuum system from two close sources, heated by an electron beam which is deflected between them. By using quartz-crystal monitors the evaporation rates are measured separately, which is usually considered to be problematical. One rate signal is used to regulate the appropriate deposition rate by controlling the emission current. The other deposition rate is regulated by using the rate signal to control the dwell-time ratio of the electron beam. With this system it is possible to deposit any composition with a good homogeneity, as in our case $\mathrm{Gd}-\mathrm{Fe}$ and $\mathrm{Gd}-\mathrm{Co}$ films.

Evaporation from two close sources by a deflected electron beam has the advantages, compared with evaporation from two electron-beam sources (Edgecumbe et al 1964), of less composition variation in the plane of the deposited film (Maissel and Glang 1965) and the possibility of preparing any composition by simply adjusting the dwell-time ratio. For a good performance it is necessary to measure the evaporation rates of the two sources separately and to control the emission current and dwell time ratio of the electron beam.

The rates are measured with Kronos quartz-crystal oscillators. For separate measurements of the two rates a plate of stainless steel is mounted between the two sources in such a way that each crystal can 'see' only one source (see figure 1). The minimum distance between plate and sourceholder should be about $4 \mathrm{~cm}$ to avoid interference with the electron beam. The distance between the crystals is also limited because of the crystal holders. From these limitations and the other dimensions given in figure 1 , it can be calculated that the length of the plate has to be at least $30 \mathrm{~cm}$ to ensure full separation of the vapour beams with respect to the crystals. The substrate of course can 'see' both sources. To avoid systematic errors, symmetry between crystals and substrate is necessary and therefore their deposition angles must be the same; in our case this angle was about $5^{\circ}$. The monitors used were the Kronos QM-331 with rate output and the Balzers QSG 101 monitor, modified for a Kronos $5 \mathrm{MHz}$ quartz-crystal oscillator, in combination with the Balzers rate monitor QRG 201B, which also contains a proportional-integral-differential (PID) regulator for rate control (see figure 2). The output of the latter is directly connected to the emission rate control unit of the power supply for the electron gun (Leybold Heraeus ESV 6). In this way rate $R_{1}$ is regulated by controlling the total emission power independent of the dwell-time ratio.

The other rate $R_{2}$ can be regulated by controlling the dwell-time ratio, i.e. the division of the total beam power on both sources. Therefore (see figure 2 ) the rate $R_{2}$ is compared with the desired value, represented by an adjustable

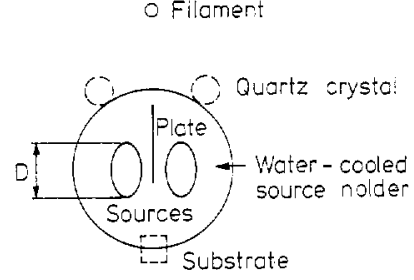

(a)
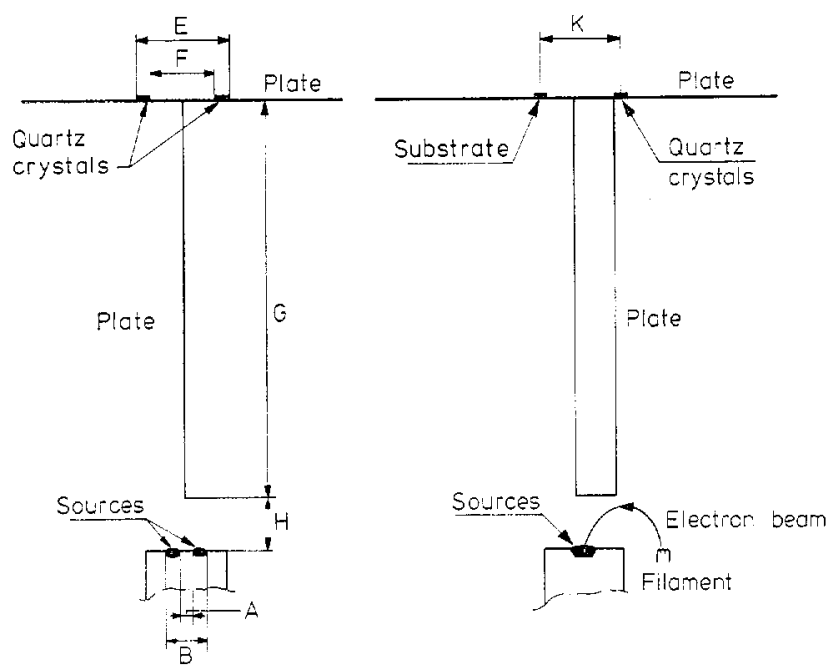

$(D)$

(c)

Figure 1 The arrangement of the gun, crystals, substrate and separation plate: $(a)$ top view, $(b)$ front view, $(c)$ side view. The dimensions are: $A=8 \mathrm{~mm}, B=32 \mathrm{~mm}, C=40 \mathrm{~mm}$, $\mathrm{D}=20 \mathrm{~mm}, \mathrm{E}=50 \mathrm{~mm}, \mathrm{~F}=30 \mathrm{~mm}, \mathrm{G}=300 \mathrm{~mm}$ (minimum), $\mathrm{H}=40 \mathrm{~mm}, \mathrm{~K}=60 \mathrm{~mm}$.

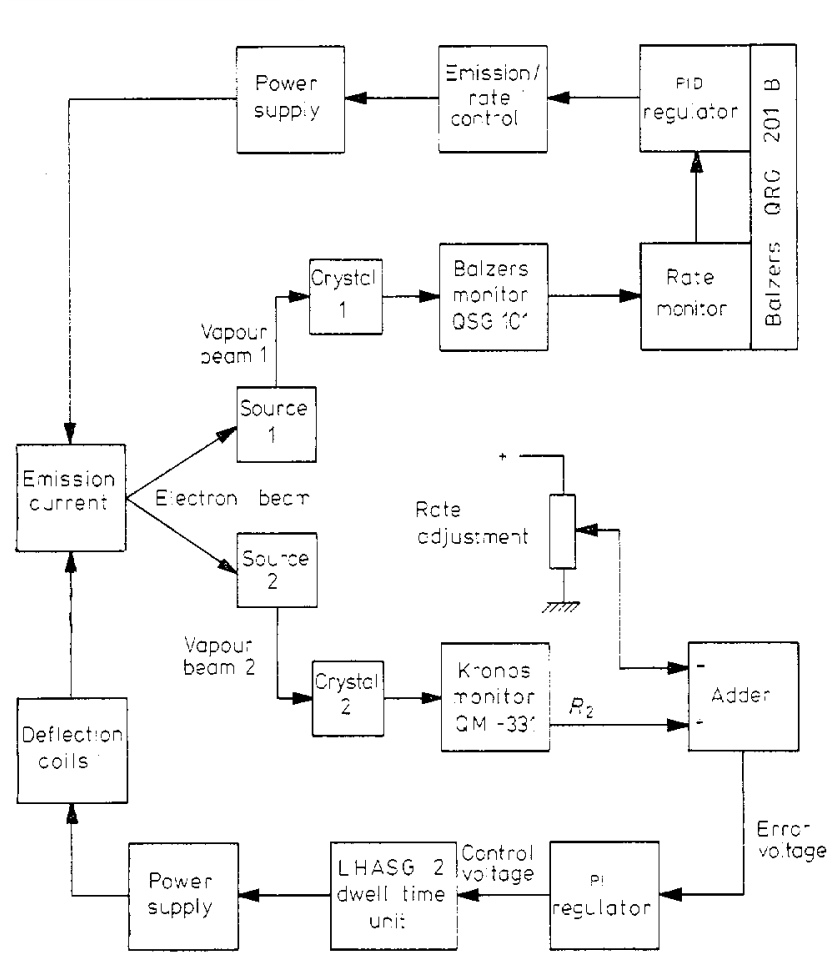

Figure 2 The complete rate control system; the manual adjustment for the dwell time ratio is incorporated in the LH ASG1. 


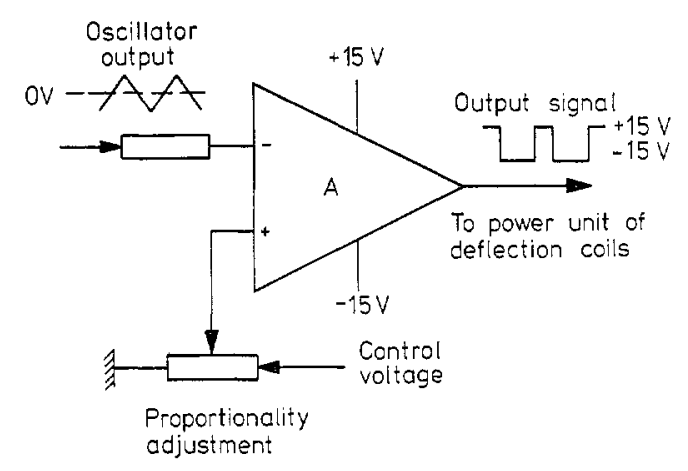

Figure 3 The trigger circuit for achieving the adjustable square wave for the dwell time ratio of the electron beam. A, operational amplifier.

voltage. The difference is an error voltage which is amplified by a PI regulator. The output of the latter is connected to the positive input of the trigger in the Leybold Heraeus dwell-time ratio unit ASG1 (see figure 3) where it is compared with a triangular signal. The output signal is a square wave which by means of a power unit drives the deflection coils of the electron beam.

The method of Ziegler and Nichols (1942) is useful for a rough determination of the gain $K$ and the integration constant $T$ of the PI regulator. In essence, without the integration constant the gain $K_{\mathrm{u}}$ is determined at which oscillation takes place with period $T_{\mathrm{u}}$ and $K$ is taken as $0.45 K_{\mathrm{u}}$ and $T$ as $0.5 T_{\mathrm{u}}$. Of course, these parameters depend on many variables, of which the material used is the most important one. It is our experience that metals like iron, cobalt, titanium and gadolinium have evaporation characteristics that are stable enough to regulate the rates within a few per cent, but in the case of unstable materials, or whatever the origin of the instability may be, oscillation of the control systems, which influence each other indirectly, will greatly reduce the efficiency. In this case it is better to use only the rate emission control and to regulate the dwell time ratio manually.

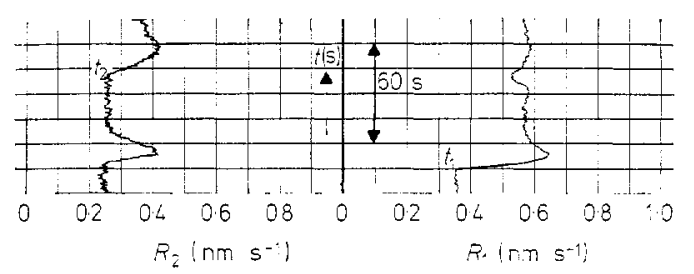

Figure 4 The rate-time dependences of the rates $R_{1}$ and $R_{2}$ subjected to a sudden increase at times $t_{1}$ and $t_{2}$.

As an example of the system behaviour the rate-time dependences of the deposition rates of two titanium sources are given (figure 4). At time $t_{1}$ rate $R_{1}$ and at $t_{2}$ rate $R_{2}$ are subjected to a sudden increase and the step response is recorded. Typical values used are $0.5-1.0 \mathrm{~nm} \mathrm{~s}^{-1}$ for the deposition rates, $10 \mathrm{~Hz}$ for the jumping frequency of the electron beam and about $2 \mathrm{~kW}$ for the total beam power. The jumping frequency must be high enough to prevent rate modulation caused by thermal relaxation of the sources and low enough to preserve the squareness of the electron deflection.

\section{Acknowledgment}

We would like to thank Professor Dr G H Jonker for his helpful discussions.

\section{References}

Edgecumbe J, Rosner L G and Andersen D E 1964

Preparation on properties of thin film hard superconductors J. Appl. Phys. 352198

Maissel L I and Glang R 1965 Handbook of thin film technology (New York: McGraw-Hill) pp 1.55-1.57

Ziegler J G and Nichols N B 1942 Optimum settings for automatic controllers

Trans. ASME 64 759-68 